\title{
Enhanced Demand and Capacity Balancing based on Alternative Trajectory Options and Traffic Volume Hotspot Detection
}

\author{
Marc Melgosa, Xavier Prats \\ Technical University of Catalonia \\ Castelldefels, Spain
}

\author{
Yan $\mathrm{Xu}$ \\ Cranfield University \\ Cranfield, United Kingdom
}

\author{
Luis Delgado \\ University of Westminster \\ London, United Kingdom
}

\begin{abstract}
Nowadays, regulations in Europe are applied at traffic volume (TV) level consisting in a reference location, i.e. a sector or an airport, and in some traffic flows, which act as directional traffic filters. This paper presents an enhanced demand and capacity balance (EDCB) formulation based on constrained capacities at traffic volume level. In addition, this approach considers alternative trajectories in order to capture the user driven preferences under the trajectory based operations scope. In fact, these alternative trajectories are assumed to be generated by the airspace users for those flights that cross regulated traffic volumes, where the demand is above the capacity. For every regulated trajectory the network manager requests two additional alternative trajectories to the airspace users, one for avoiding the regulated traffic volumes laterally and another for avoiding it vertically. This paper considers that the network manager allows more flexibility for the new alternative trajectories by removing restrictions in the Route Availability Document (RAD). All the regulated trajectories (and their alternatives) are considered together by the EDCB model in order to perform a centralised optimisation minimising the the cost deviation with respect to the initial traffic situation, considering fuel consumption, route charges and cost of delay. The EDCB model, based on Mixed-Integer Linear Programming (MILP), manages to balance the network applying ground delay, using alternative trajectories or both. A full day scenario over the ECAC area is simulated. The regulated traffic volumes are identified using historical data (based on 28th July of 2016) and the results show that the EDCB could reduce the minutes of delay by $70 \%$. The cost of the regulations is reduced by $11.7 \%$, due to the reduction of the delay, but also because of the savings in terms of fuel and route charges derived from alternative trajectories.

Index Terms-Demand and capacity balance, hotspot, alternative trajectory, traffic volume.
\end{abstract}

\section{NOMENCLATURE}

$$
\begin{aligned}
& f \in \mathcal{F} \\
& m \in \mathcal{M}_{f} \\
& l \in \mathcal{P}_{m} \\
& t \in T \\
& \tau \in \mathcal{T} \\
& P(m, i) \\
& r_{m}^{l} \\
& T_{m}^{l} \\
& C_{l}(\tau) \\
& \delta_{m} \\
& \alpha_{m}
\end{aligned}
$$

set of regulated flights

set of trajectories $m$ for flight $f$

set of traffic volumes that $m$ traverses

set of time moments

set of periods for unit capacity

traffic volume $i$ for trajectory $m\left(i \in \mathcal{P}_{m}\right)$

the initially scheduled time of $m$ at $l$

$\left[r_{m}^{l}, r_{m}^{l}+e_{m}^{l}\right]$, the feasible time window for $m$ at $l$ the remaining sector capacity of the traffic

volume $l$ in $\tau$

the fuel cost in $€ / \mathrm{Kg}$ for $m$

the ground delay cost in $€ / \mathrm{min}$ for $m$

\section{INTRODUCTION}

The air transportation network is limited by operational constraints that bring to maximum declared capacities for airports and airspace sectors [1]. Demand and Capacity Balancing (DCB) models aim at maintaining the traffic demand below the capacity when the network is overloaded, fact that happens when the demand is too high or when capacity is reduced (as for example due to disruptive weather or Air Traffic Control limitations) and leads to the application of Traffic Management Initiatives (TMI). According with [2], the European air traffic increased by $3.8 \%$ in 2018 and the network generated a total of 19.1 million minutes of en-route delay (a 105\% more with respect 2017). For this reason, it is important to improve DCB models.

In Europe, Air Traffic Flow Management (ATFM) is provided by the Network Manager (NM). The ATFM slot allocation is done by the Computer-Assisted Slot Allocation (CASA) algorithm [3], based on rules agreed and accepted by relevant Air Traffic Management (ATM) stakeholders.

In the United States, Ground Delay Programs (GDPs) and Airspace Flow Programs (AFPs) are the most commontly used TMIs. GDPs assign departure delays in order to manage the demand at the arrival airport. On the other hand, AFPs focus on constrained en-route elements, i.e., Flow Constrained Areas (FCA) [4]. The AFPs offer two different solutions to tackle the constrained area: delaying flights or specifying alternative routes that bypass the capacity-constrained area. Then, the aircraft operator concerned can choose either alternative (reroute or the delay) [5].

The previous TMIs use a ration-by-schedule (RBS) algorithm as basis for the flight scheduling, which is accepted by the aviation community as fairness criterion [6]. However, this methodology does not take into account the airspace user (AU) preferences and could be improved to be better aligned with the new concept of operations proposed by SESAR (Single European Sky ATM Research) in Europe and NextGen (Next Generation Air Transportation System) in the United States. In this context, Collaborative Decision Making (CDM) initiatives have already been introduced for enabling the airspace user involvement and to provide more flexibility in the route selection [3]. Under this scope, in the United States, the 
GDP concept with RBS was extended with flight substitutions and the cancellation-compression algorithm, allowing more flexibility to the airspace users for satisfying their own policies [7]. Regarding the AFPs, the CDM concept was introduced with the Collaborative Trajectory Options Program (CTOP), where airspace user is able to communicate their preferences in terms of route selection using Trajectory Options Sets (TOSs) [8].

Although current version of CTOP implements RBS, alternative Mixed-Integer Linear Program (MILP) formulations were introduced for instance in [9], [10], or [11]. Similar to the principle of CTOP, a more user-driven approach was introduced in [12], where the alternative trajectories were generated by AUs based on some information shared from the ATFM unit. Different types of delay initiatives were also allowed, such as ground holding, airborne holding, linear holding, as well as delay recovery, so as to reflect AUs' specific preferences.

All previous works considered capacities at sector level. However, regulations in Europe are applied at traffic volume level consisting in a reference location, i.e., a sector or airport, and in some traffic flows, which act as directional traffic filters [13]. In this paper, an alternative to the previous work in [12] is proposed, introducing a novel methodology that uses the regulated traffic volumes (instead of sectors) to identify the flights to be regulated. This may reduce the impact in the network proposed in previous work making it more comparable to current practice. Another contribution with respect to [12] is that this paper extends the regulated area from national scale to the whole ECAC area. For the flight planning inside this area, there is the Route Availability Document (RAD), that can be seen as a first strategic DCB measure since some restrictions can be applied in order to prevent disruptions on major traffic flows [14]. In this paper, in order to potentially compensate the adverse consequences that a regulation may entail to the airspace users, the restrictions of this documents are not enforced when an alternative trajectory is requested to the AUs.

This paper presents a new EDCB model that: 1) uses historical regulation in order to identify the hotspots; 2) allows the airspace users to provide alternative trajectories in case of regulation (some relaxation in terms of RAD restrictions are allowed); 3) considers ground delay or reroute options; and 4) optimises the traffic flow to obtain the best global cost option.

\section{THE USE OF TRAFFIC VOLUMES}

In realistic operations, a certain amount of capacity overloads are usually allowed. Several reasons could explain this phenomena: the lack of initial schedules for non-planed flights, the use of entry rate for assessing the demand without considering the occupancy, a conservative way for estimating the capacity and the complexity of traffic patterns, etc. In this paper, the limitation due to traffic patterns is eliminated by changing the airspace unit used in the hotspot detection from sector to traffic volume. In addition, the real regulation list is also used in order to take into account other capacity overload allowance reasons.

Currently, the regulations in Europe are applied to traffic volumes. In fact, every traffic volume is related with a reference location, that can be a sector, a collapsed sector or an airport. In addition, one traffic volume is defined by a set of included and excluded flows, what can be understood as a directional filter. For example, Fig. 1 shows a sector where there are three major flows, A-E, B-D, and C-F. It seems logical that the intersection between the flows A-E and B-D can create some capacity constraint. Thus, a regulation over a TV related with this sector and including the flows A-E and B-D and excluding D-F may be applied.

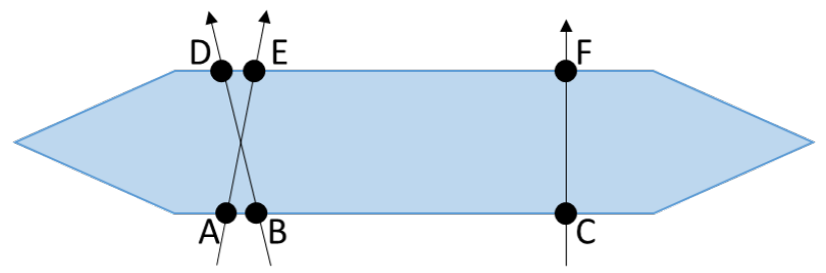

Fig. 1. Illustrative example of traffic volume

There is also the case where one traffic volume has not any included/excluded flows. In this case, all flights crossing the associated area are considered and there are not differences between sector and traffic volume. Furthermore, it is important to note that some regulations are applied to traffic volumes that are not active. It means, that the regulated traffic volume is related to a sector that is not used. This situation can happen, for example, to avoid an airspace zone due to bad weather conditions or to force an ATC routing deviation. In order to consider this situation, the opening scheme is not considered, meaning that the network manager will continuously monitor the traffic.

Furthermore, in order to consider the allowed capacity overloads, the declared capacity of the traffic volumes per every time period is updated with the demand when the demand is higher than the capacity and the traffic volume is not regulated in such time period. In addition, for the non-active traffic volumes there are not declared capacities available. In this work, a minimum of 20 operations per hour is assumed in these situations.

\section{FRAMEWORK}

The framework implemented in this paper is presented in Fig. 2. The starting point is the initial traffic list, consisting in the last filled flight plans reconstructed to 4D trajectories. In this paper, these trajectories represent the preferred ones by the airspace users, i.e. the first submitted shared business trajectory (SBT) using SESAR's terminology. This initial traffic data, together with the regulation list, as well with the geographic location of the traffic volumes, are used by the "Identification of regulated flights in TV" module in order to identify those flights that should be regulated. In addition, the flights crossing a regulated traffic volume (non related with 
any airport) will be also candidates for requesting alternative trajectories. This part of the framework emulates the first stage of the network manager work, where the hotspots are identified at traffic volume level and and the alternative trajectories set is requested to the airspace users. Thus, the output of this module is the affected traffic list and the lateral/vertical avoidance requirements for the airspace users in case they want to offer an alternative trajectory.

The next module of the framework is the "Trajectory optimisation", that emulates the behaviour of the airspace users. This module finds a new trajectory that avoids the regulated traffic volumes using the available route network. This submodule is based on DYNAMO [15] and finds out a new optimal trajectory in terms of flight operating costs. In fact, the alternative trajectory provided minimises a compound cost function $J_{m}$ over the whole time window $\left[t_{0}, t_{f}\right]$ and flight path $\left[x_{0}, x_{f}\right]$ as follows:

$J_{m}=\int_{t_{0}}^{t_{f}} \delta_{m}\left(\dot{F}_{m}(t)+C I_{m}\right) \mathrm{d} t+\frac{\sqrt{M T O W_{m}}}{5 \cdot 10^{5} \sqrt{2}} \int_{x_{0}}^{x_{f}} k(x) \mathrm{d} x$

where $\dot{F}_{m}(t)$ is the fuel flow, $C I_{m}$ is the Cost Index, MTOW is the Maximum Take-Off Weight and $k(x)$ is the route charges unit rate combined to reflect airspace users' direct operating costs.

It is important to note that, currently, the route charges are paid based on the filled flight plan. This paper considers to recalculate the route charges for the alternative trajectories in order to emulate a futuristic scenario where the presented EDCB is used and the route charges are always considered. In fact, the DCB problem may be solved by alternative trajectories and the workload of the Area Control Centres (ACCs) close to the hotspots may vary. In addition, the route charges are calculated using the real distance flown inside an area instead of using the great circle distance between the entry and exit point of such area as it is currently applied.

Here, it is important to mention that the alternative trajectories are allowed to disregard the Route Availability Document (RAD). In other words, the current structured route concept of operation is used, but the segment altitude restrictions, segment constraints related with the origin and destination airports or waypoints, etc. are not considered. This action tries to compensate the adverse consequences that a regulation may entail to the airspace users. In fact, some alternative trajectories may be more optimal than the initial ones, since the latter trajectories could have been planed by observing the RAD.

After the alternative trajectories generation, the alternative trajectories set is obtained consisting in the initial trajectories and the alternative ones for the required flights (it is important to note that the creation of a new trajectory does not replace the original one). An example of alternative trajectory set for one flight from Manchester (EGCC) to Barcelona (LEBL) is shown in Fig. 3. In Fig. 3(a) the original trajectory (in red) and the lateral avoidance trajectory (in green) are shown. Fig. 3(b) presents the comparison between the original trajectory (in red) and the vertical avoidance trajectory (in green).
After the provision of the alternative trajectories set by the AUs, the cost of all the trajectories shall be evaluated in terms of fuel consumption and route charges. This information is estimated by the network manager. However, in a futuristic and collaborative scenario, such information may be provided by the AUs.

Once the airspace users have provided all the trajectories and the fuel cost and route charges are estimated, the module "MILP Network optimiser" is in charge of optimising the network selecting which is the best trajectory to be performed per every flight and the amount of ground delay assigned. The declared capacity from the traffic volume definition is used to avoid overloads. As this module is the main contribution of this paper, the next section explains the details and formulation of this optimisation problem.

\section{DEMAND AND CAPACITY BALANCING}

The EDCB network optimiser module is formulated as a MILP problem, where the balancing between the demand and the capacity is conducted by selecting one of the available trajectories per flight, applying ground delay or both. The module aims at minimising the cost of the regulations, consisting in the addition of the fuel cost, the delay cost and the route charges. The following subsections aim at presenting the problem to be considered, the used objective function and the set of constraints applied.

\section{A. Problem statement}

The problem consists into manage the demand below the capacity for all the regulated traffic volumes and for the active traffic volumes linked with one airport inside the network manager area. Flights departing from other airports can not be delayed and for this reason are excluded. As it has been introduced before, there are two decisions to be taken: 1) Which trajectory is used per each flight? 2) How much delay is applied? In order to give answer to such questions, two decision variables are introduced:

(1) Decision variable for trajectory options:

$z_{m}^{f}= \begin{cases}1, & \text { if trajectory } m \text { is chosen for flight } f \\ 0, & \text { otherwise }\end{cases}$

(2) Decision variable for delay assignment:

$x_{m, t}^{l}= \begin{cases}1, & \text { if trajectory } m \text { arrives at traffic volume } l \text { by time } t \\ 0, & \text { otherwise }\end{cases}$

Note that, according to [10], the use of "by" time provides faster solution time than 'at" time. However, the "at" time expressions can be easily found by $\left(x_{m, t}^{l}-x_{m, t-1}^{l}\right)$, for all $m, l$ and $t$ in the respective sets.

\section{B. Objective function}

It may seem that the initially scheduled trajectory may be the preferred one by the airspace users, but it is important to recall that the flight plan trajectory has been planned under the RAD restrictions. In addition, the trajectory optimisation tool 


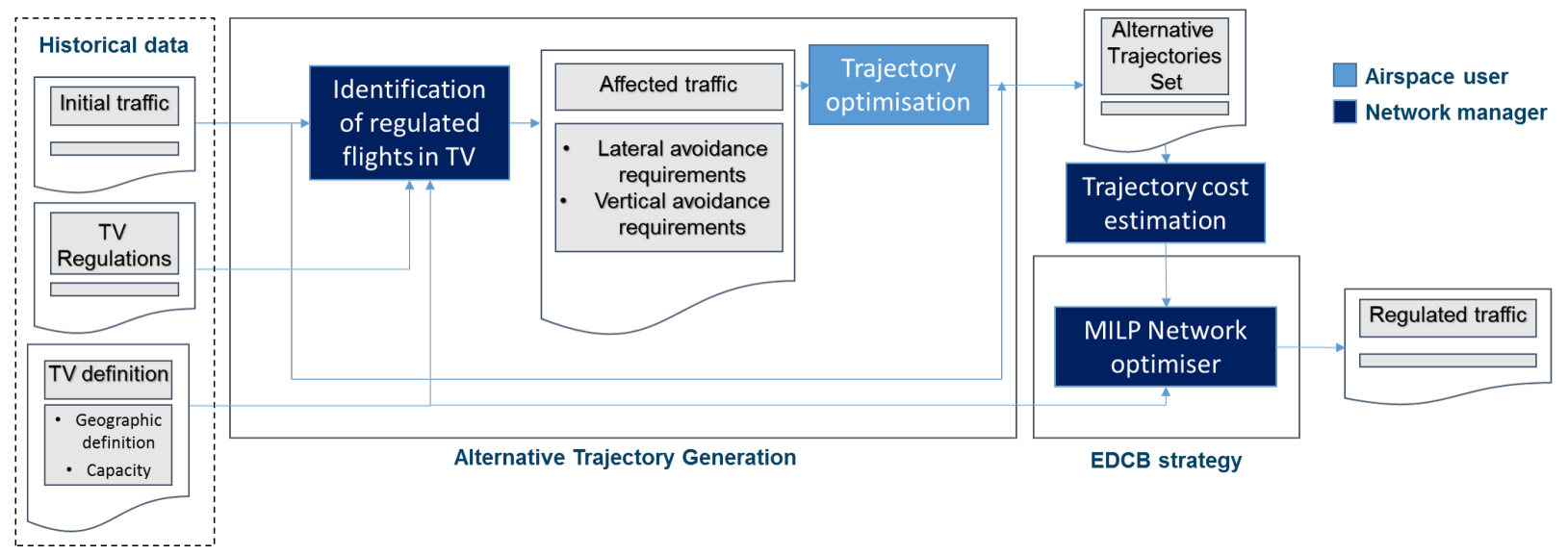

Fig. 2. EDCB framework

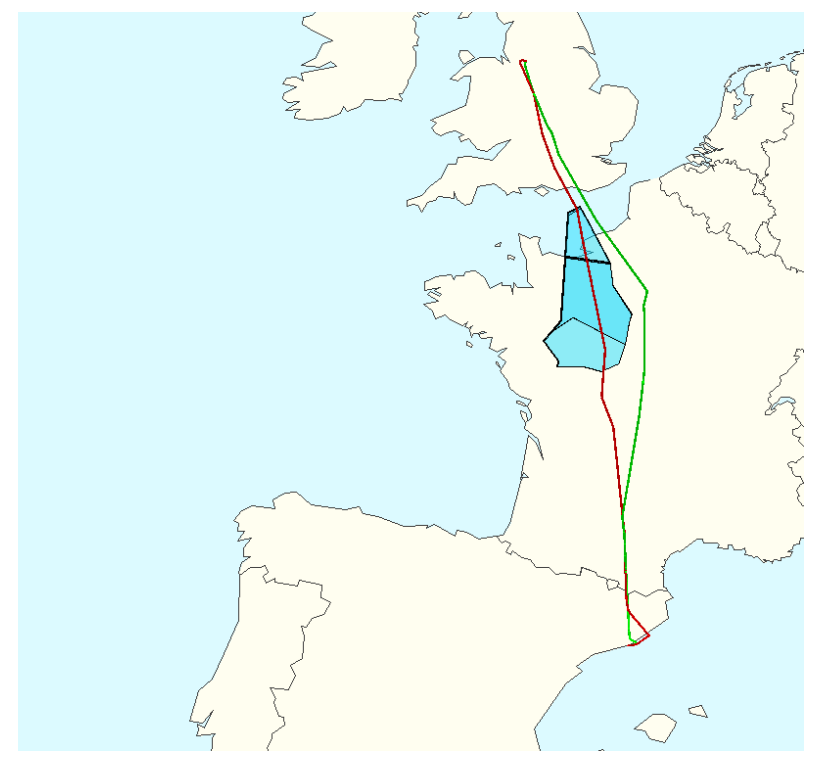

(a) Lateral (green) VS original (red) trajectory

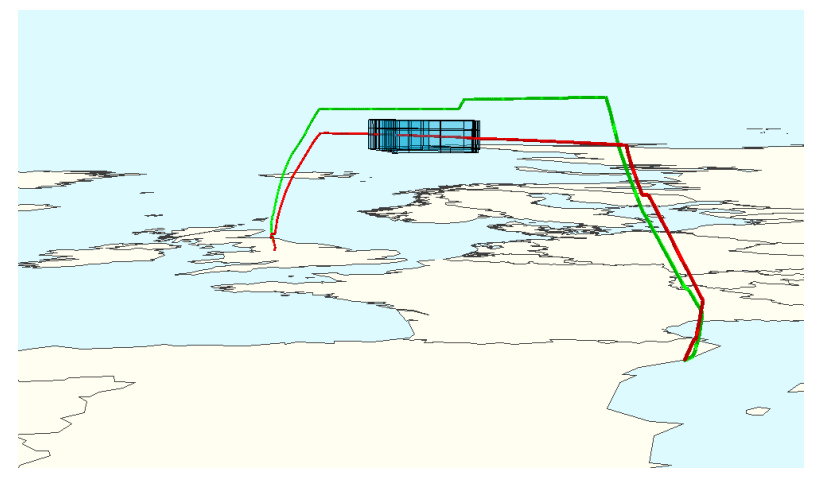

(b) Vertical (green) VS original (red) trajectory

Fig. 3. Example of TOS

DYNAMO does not optimise laterally the Standard Instrument Departures (SIDs) and the Standard Instrumental Arrivals (STARs) procedures. Hence, some alternative trajectories may result in less cost than the initial ones, so they may be the preferred by the airspace users. The objective function, however, minimises the difference between the total cost (considering the extra fuel consumption, extra route charges and delay costs) of the selected trajectory with respect to the initial trajectory:

$$
\min J=\min \left(C_{\Delta F}+C_{D}+C_{\Delta R}\right)
$$

The total extra fuel consumptions $C_{\Delta F}$ can be obtained as follows:

$$
C_{\Delta F}=\sum_{f \in \mathcal{F}} \sum_{m \in \mathcal{M}_{f}} \delta_{m} \cdot\left(F_{m}-F_{f}\right) \cdot z_{m}^{f}
$$

where $\delta_{m}$ is the fuel cost for $m, F_{m}$ is the total fuel consumed by trajectory $m$ and $F_{f}$ is the total fuel consumed by the initial trajectory of flight $f . m \in \mathcal{M}_{f}$ means that $m$ is one of the trajectory options of flight $f$. Similarly, the extra route charges $C_{\Delta R}$ are denoted as:

$$
C_{\Delta R}=\sum_{f \in \mathcal{F}} \sum_{m \in \mathcal{M}_{f}}\left(R_{m}-R_{f}\right) \cdot z_{m}^{f}
$$

where $R_{m}$ is the total route charge of trajectory $m$ and $R_{f}$ is the total route charge of the initial trajectory of flight $f$.

The extra delay time is basically derived from the ground holding. Thus, it can be expressed as:

$$
C_{D}=\sum_{f \in \mathcal{F}} \sum_{m \in \mathcal{M}_{f}} \alpha_{m} \cdot G H_{m}
$$

where $\alpha_{m}$ is the ground delay cost for $m$ and $G H_{m}$ is the ground holding time of the trajectory $m$ :

$$
G H_{m}=\sum_{t \in T_{m}^{l}, l=P(m, 1)}\left(t-r_{m}^{l}\right) \cdot\left(x_{m, t}^{l}-x_{m, t-1}^{l}\right)
$$

The objective function presented aims at minimising the global cost between the regulated flights. 


\section{Constraints}

This subsection lists and describes all the constraints applied to the EDCB model presented:

- One trajectory, i.e. the initial or one of the alternatives must be selected.

$$
\sum_{m \in \mathcal{M}_{f}} z_{m}^{f}=1 \quad \forall f \in \mathcal{F}
$$

- Constraints for applying the "by" time technique

$$
\begin{array}{r}
x_{m, \underline{T}_{m}^{l}-1}^{l}=0 \quad \forall f \in \mathcal{F}, \forall m \in \mathcal{M}_{f}, \forall l \in \mathcal{P}_{m} \\
x_{m, \bar{T}_{m}^{l}}^{l}=z_{m}^{f} \quad \forall f \in \mathcal{F}, \forall m \in \mathcal{M}_{f}, \forall l \in \mathcal{P}_{m} \\
x_{m, t}^{l}-x_{m, t-1}^{l} \geq 0 \quad \forall f \in \mathcal{F}, \forall m \in \mathcal{M}_{f}, \forall l \in \mathcal{P}_{m}, \\
\forall t \in T_{m}^{l}
\end{array}
$$

Constraint 9 forces that all the decision variables are equal to zero when the trajectory is not selected. In addition, $T_{m}^{l}$ is the time resolution of the model.

- Only ground holing is allowed.

$$
x_{m, t+r_{m}^{l+1}-r_{m}^{l}}^{l+1}-x_{m, t}^{l} \geq 0 \quad \forall f \in \mathcal{F}, \forall m \in \mathcal{M}_{f}, \forall l \in \mathcal{P}_{m},
$$

Where $l+1$ is the next traffic volume that the trajectory $f$ crosses after $l$.

- The demand can not exceed the remaining capacity of any traffic volume

$$
\begin{array}{r}
\sum_{m \in \mathcal{M}_{f}} \sum_{l \in \mathcal{P}_{m}} \sum_{t \in T_{m}^{l} \cap T(\tau)}\left(x_{m, t}^{l}-x_{m, t-1}^{l}\right) \leq C_{l}(\tau) \\
\forall f \in \mathcal{F} \quad \forall \tau \in \mathcal{T}
\end{array}
$$

Note that $C_{l}$ refers to remaining capacity. As the EDCB problem takes into consideration only the regulated flights, the non-regulated traffic demand $\left(N D_{l}\right)$ should be subtracted from the declared capacity $\left(D C_{l}\right)$ :

$$
C_{l}(\tau)=D C_{l}(\tau)-N D_{l}(\tau) \quad \forall l \in \mathcal{P}_{m}, \forall \tau \in \mathcal{T}
$$

\section{CASE STUdY AND RESUlts}

\section{A. Workflow setup}

Regarding the workability of the framework, the initial traffic, the regulation list and the traffic volume definition are obtained from the DDR2 database. The identification of regulated flights in a TV is done using NEST v1.6.6, which allows to get the entry list for every traffic volume (containing the flight identification code together with the entry time to the traffic volumes). The trajectory optimisation tool is based on DYNAMO, that needs the initial mass and the cost index (CI) of the aircraft as inputs for the trajectory optimisation.
Those parameters are estimated using the initial trajectories and a model that relies on the aircraft performance models provided by the Base of Aircraft Data (BADA) v4 and needs to set up the aircraft mass at the end of the flights [16]. For the purposes of this paper, such final mass is assumed to be the $90 \%$ of the Maximum Landing Weight (MLW). The alternative trajectories are created using the same mass and cost index estimated for the original flights with the required constraints for avoiding the traffic volumes (provided by the network manager).

For the ECDB startegy, in line with the ATFM costs in Europe proposed in [17], the cost assumed for the ground delay and the fuel is $81 € / \mathrm{min}$ and $0.6 € / \mathrm{kg}$, respectively. For this experiment, cost of the delay and the fuel is also assumed the same for all flights.

\section{B. Experimental setup}

This paper analyses a 24 hour scenario based on the European Civil Aviation Conference (ECAC) area. The time resolution $\left(T_{m}^{l}\right)$ for the evaluation of the formulation presented in Section IV is set to five minutes. The capacity of the traffic volumes is evaluated for periods of 20 minutes.

The day of study is the 28th July of 2016 and the traffic sample contains 32,960 flights crossing the ECAC zone. As the experiment is applied to 24 hours and the capacity is evaluated in periods of 20 minutes, the experiment is divided in 72 periods. During this day, there were 179 regulations. However, only the regulations starting and ending in the same day are considered. It means that the scenario takes into consideration 175 regulations affecting a total of 120 traffic volumes (see Fig. 4 to see all the regulated traffic volumes), meaning that some traffic volumes were affected with more than one regulation. Nevertheless, this experiment wants to consider the regulated traffic volumes, as well as the active traffic volumes related with an airport inside the network manager area (equivalent to the ECAC area). The objective is to avoid overloads in the regulated traffic volumes but not creating overloads in the airports as a consequence of the ground holdings. Thus, this experiment monitors a total of 545 traffic volumes.

Regarding the affected flights, a total of 7,168 flights crossed at least one traffic volume during the time they were under a regulation. Nevertheless, only the flights departing from airports inside the network manager area are subjected to regulations, so the DCB problem is applied to only 6,386 flights. The rest of the flights satisfy the flight plan and no delay is applied to them.

Note that if the regulated traffic volume is linked with the origin or destination airport, it can not be avoided by the use of alternative trajectories. Thus, from the 6,386 regulated flights, only 4,931 could submit alternative trajectories to avoid the regulated airspace. For those flights, the network manager may ask to the airspace users to provide new alternative trajectories. In this experiment, two alternative trajectories are requested per flight, avoiding the crossing regulated traffic volumes (non related with any airport) vertically and horizontally. However, 


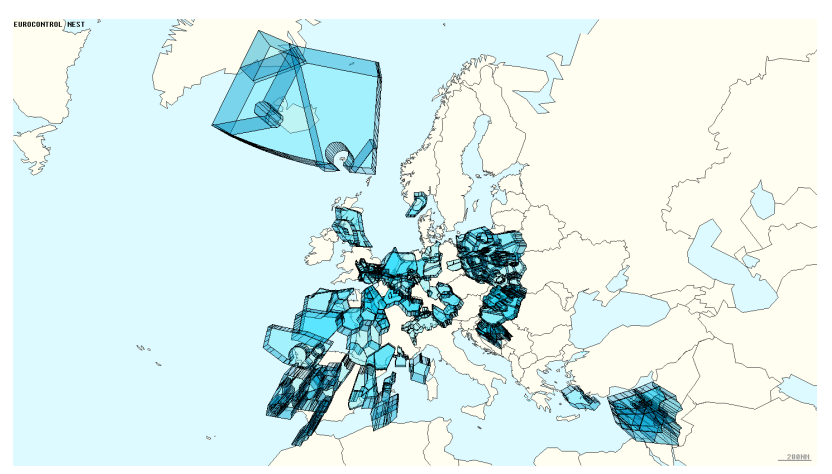

Fig. 4. Regulations of 28th July of 2016

TABLE I

SUMMARY OF THE EXPERIMENT DATA

\begin{tabular}{ll}
\multicolumn{2}{c}{ Traffic data } \\
Flights crossing ECAC & 32,960 \\
Flights crossing a regulated TV & 7,168 \\
Regulated flights & 6,386 \\
Lateral trajectories & 4,091 \\
Vertical trajectories & 3,770 \\
\multicolumn{1}{c}{ Constrained airspace } & \\
Number of regulations & 175 \\
Regulated TV & 120 \\
Considered TV & 545
\end{tabular}

some of these alternative trajectories are not feasible. There could be different reasons, such as the regulated traffic volume is very close to the origin or destination airport and can not be avoided; or the current route structure do not allow to connect the origin and destination with the avoidance restrictions. In addition, a current limitation of DYNAMO is that it can be used only for jet aircraft. It means that alternative trajectories for propeller aircraft can not be simulated.

The final number of alternative trajectories provided is 4,091 (63.9\% of the regulated flights) and 3,770 (59\% of the regulated flights), avoiding the regulated traffic volumes laterally and vertically, respectively. This data is summarized in Table I.

\section{Results}

It is important to describe a baseline scenario in order to compare the results. In this case, the baseline scenario is obtained from historical data from the DDR2 database, where the DCB problem is tackled using the Computer Assisted Slot Allocation (CASA) delay algorithm (based on Rationby-Schedule), which is highly accepted as a fair solution. In this case, a total of 3,518 flights were delayed, accumulating a delay of 58,622 minutes. Analysing only the delayed flights, the average delay is 16.66 minutes, being the maximum delay applied to one flight 139 minutes. In this case, the median is 14 minutes so the half of the delayed flights has 14 minutes of delay or less. The standard deviation of the delay is 11.50 minutes (see Table II).

The same problem solved with the EDCB methodology presented in this paper results in a total delay of 17,575 minutes applied to 855 flights. Now, the average delay is
TABLE II

SUMMARY OF THE RESULTS

\begin{tabular}{|c|c|c|c|c|}
\hline & & CASA & EDCB & $\Delta$ \\
\hline \multirow{4}{*}{$\vec{z}$} & Total regulation cost $[€]$ & $40,732,688$ & $35,979,664$ & $-11.7 \%$ \\
\hline & Fuel cost $[€]$ & $28,611,400$ & $27,388,050$ & $-4.3 \%$ \\
\hline & Delay cost $[€]$ & $4,748,382$ & $1,423,575$ & $-70.0 \%$ \\
\hline & Route Charges [€] & $7,372,907$ & $7,168,040$ & $-2.8 \%$ \\
\hline \multirow{6}{*}{ 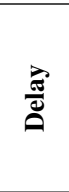 } & Total delay [min] & 58,622 & 17,575 & $-70.0 \%$ \\
\hline & Delayed flights & 3,518 & 855 & $-75.7 \%$ \\
\hline & Max. delay [min] & 139 & 355 & $155.4 \%$ \\
\hline & Average delay [min] & 16.66 & 20.56 & $23.4 \%$ \\
\hline & Median delay [min] & 14.00 & 10.00 & $-28.6 \%$ \\
\hline & Standard dev. [min] & 11.5 & 32.87 & $185.8 \%$ \\
\hline \multirow{3}{*}{ 魚冚 } & Total fuel [Tn] & 47,686 & 45,647 & $-4.3 \%$ \\
\hline & Total distance [NM] & $6,237,291$ & $6,212,292$ & $-0.4 \%$ \\
\hline & Total trip time [min] & 896,153 & 884,732 & $-1.3 \%$ \\
\hline \multirow{3}{*}{ 承 } & Original & 6,389 & 3,382 & - \\
\hline & Lateral & 0 & 1,900 & - \\
\hline & Vertical & 0 & 1,104 & - \\
\hline
\end{tabular}

20.56 minutes and the maximum delay is 355 minutes. These values are higher than using CASA, but it is important to look at the value of the median. Here, the median is 10 minutes, what means that the half of the delayed flights are delayed 10 minutes or less (less than using CASA). The big standard deviation, i.e. 32.87 , matches with the big number of the maximum delay and the higher average value. Thus, the EDCB presented allows to reduce the total delay, but a few number of flights can accumulate a big amount of delay. It means that with EDCB the delay can be reduced but the fairness is perhaps decreased. This could be partially mitigated by including in the objective function a fairness factor (i.e., a superlinear coefficient for the cost of delay imposed on one flight) as done in [12], but a more sophisticated equilibrium mechanism in this regard is still subject to ongoing research.

As it can be seen, the number of delayed flights with EDCB represents only the $24.30 \%$ of the delayed flights number obtained with the CASA algorithm. Regarding the minutes of delay, the EDCB models gets the $30.00 \%$ of minutes obtained by the current delay methodology.

Besides, the new EDCB model introduces the use of alternative trajectories. From the 6,386 regulated flights, 3,382 flights used the original trajectory, 1,900 flights preferred to use the lateral re-route trajectory and 1,104 used the vertical rerouted trajectories. Table II also shows summary of the results in comparison with the baseline scenario.

Now, it is important to recall that the EDCB problem is solved minimising the cost of the ground holding and the difference in terms of fuel cost and route charges between the alternative trajectories and the original trajectory (see Equation 2). The total cost of the EDCB is $35,979,664 €$, what represents a reduction of $11.7 \%$ with respect the baseline scenario.

This EDCB solution allows to save 2,039 Tons of fuel ($4.38 \%$ with respect with the CASA). Different reasons can explain this saving. First, the alternative trajectories have been simulated without RAD constraints. It means, for example, that some traffic volumes may be avoided just flying at the optimal flight level. Another explanation for the savings is that, 
although one can think that the lateral avoidance trajectory will be longer than the original, it does not happen the most of the times. If fact, the results show that some lateral alternative trajectories are shorter than the initial trajectory acquiring a saving of 25,000 NM (-0.4\% respect to CASA). Fig. 5 shows an example where the alternative trajectory is shorter than the initial one, in this case by $24 \mathrm{NM}$. This distance reduction can be consequence of the exclusion of the RAD constraints, but the lack of SIDs and STARs into the alternative trajectory simulation tool can be also part of such reduction. An effect of the shorter trajectories and optimal vertical profiles is that the trip time is reduced, providing a saving of 11,421 minutes $(-1.27 \%$ compared with the baseline scenario). Table II shows the savings of the EDCB model.

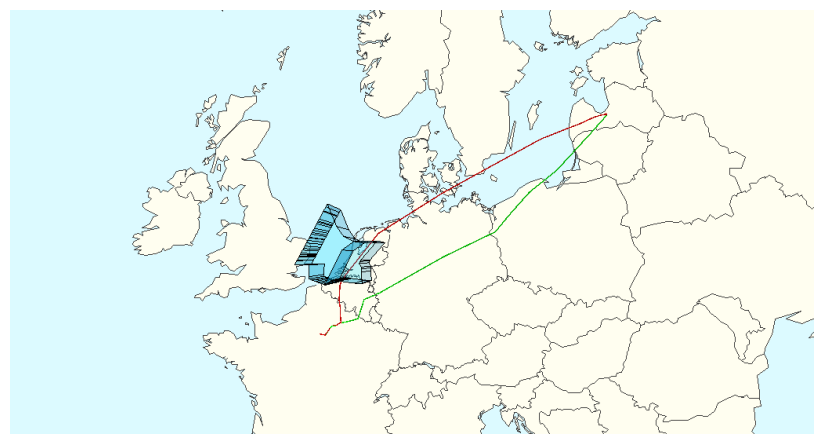

Fig. 5. Example of lateral trajectory (green) shorter than the original (red)

\section{CONCLUSION}

This paper presents a new EDCB model based on constrained capacities at traffic volume level, acting as directional traffic filters. This methodology takes into consideration the airspace users' preferences through the usage of alternative trajectories that avoids laterally or vertically the overloaded traffic volumes. Some relaxation of the RAD restrictions are allowed to the alternative trajectories in order to potentially compensate the regulation cost. Then, the network manager determines the best trajectory selection applying a global optimisation in terms of cost, including the time, the fuel and the route charges.

A 24 hour scenario based on the ECAC area has been simulated in order to compare the presented EDCB model with the current Computer Assisted Slot Allocation (CASA) model based on ration-by-schedule. Results show that the EDCB model enables a significant reduction of the delay, i.e. a reduction of the $70.0 \%$ in the delay is achieved when the demand and capacity problem is solved by the EDCB algorithm. This delay reduction, together with the saving in terms of fuel and route charges, reduces the cost of the regulations by $11.7 \%$. However, a fairness problem has been identified, so a few flights are affected by a big amount of delay.

Some open points have been identified for future work:

1) An impact study over the surrounding traffic volumes to analyse the domino effects shall be done.
2) A fairness limitations has been identified in this paper. Future work may introduce some modification into the cost function of the MILP problem in order to equilibrate the delay.

3) Although the usage of traffic volumes can be seen as an initial approximation to some complexity metrics because there are some flow identification, an evolution of the model presented to be used with a new pure complexity metric should be considered.

4) The uncertainty in the trajectories and capacities has been not used in this study. However, it should be part of the formulation because the problem shall be used in the pre-tactical phase.

5) The inclusion of the arrival delay cost in the EDCB cost function should be considered.

6) A more detailed study of the distribution of the delay over all flights shall be conduced.

\section{REFERENCES}

[1] M. Ball, C. Barnhart, G. Nemhauser, and A. Odoni, "Air transportation: Irregular operations and control," Handbooks in Operations Research and Management Science, vol. 14, pp. 1-67, 2007.

[2] "2018's air traffic in a nutshel," https://www.eurocontrol.int/news/2018air-traffic, accessed: 2019-02-20.

[3] EUROCONTROL, "ATFCM operations manual - network operations handbook," Tech. Rep. Ed. 21.0, 2017.

[4] FAA, "Traffic Flow Management in the National Airspace System," Federal Aviation Administration, Tech. Rep. FAA-2009-AJN-251, 2009.

[5] N. V. Pourtaklo and M. Ball, "Equitable allocation of enroute airspace resources," in Proceedings of the 8th USA/Europe ATM R\&D Seminar, Napa, CA, US, 2009.

[6] C. Brinton, S. Atkins, L. Cook, S. Lent, and T. Prevost, "Ration by schedule for airport arrival and departure planning and scheduling," in 2010 Integrated Communications, Navigation, and Surveillance Conference Proceedings, May 2010, pp. I3-1-I3-9.

[7] M. O. Ball, R. Hoffman, D. Lovell, and A. Mukherjee, "Response mechanisms for dynamic air traffic flow management," in Proceedings of the 6th USA/Europe ATM R\&D Seminar, Baltimore. US, 2005.

[8] FAA, "Collaborative Trajectory Options Program (CTOP): Document Information." Federal Aviation Administration, Tech. Rep. AC 90-115, 2014.

[9] O. Rodionova, H. Arneson, B. Sridhar, and A. Evans, "Efficient trajectory options allocation for the collaborative trajectory options program," in Proceedings of the 36th IEEE/AIAA Digital Avionics Systems Conference (DASC), St. Petersburg, FL, US, Sept 2017, pp. 1-10.

[10] D. Bertsimas and S. S. Patterson, "The air traffic flow management problem with enroute capacities," Operations research, vol. 46, no. 3, pp. 406-422, 1998.

[11] G. Lulli and A. Odoni, "The European air traffic flow management problem," Transportation Science, vol. 41, no. 4, pp. 431-443, 2007.

[12] Y. Xu, R. Dalmau, M. Melgosa, A. de Montlaur, and X. Prats, "Alternative trajectories for delay reduction in demand and capacity balancing," in Proceedings of the 8th International Conference for Research in Air Transportation (ICRAT), Castelldefels, Spain, 2018.

[13] EUROCONTROL, "Nest user guide 1.6," 2019.

[14] — , "Rad - route availability document," 2019.

[15] R. Dalmau, M. Melgosa, S. Vilardaga, and X. Prats, "A fast and flexible aircraft trajectory predictor and optimiser for atm research applications," in Proceedings of the 8th International Conference for Research in Air Transportation (ICRAT), Castelldefels, Spain, 2018.

[16] R. Dalmau, X. Prats, A. Ramonjoan, and S. Soley, "Estimating fuel consumption from radar tracks: A validation exercise using FDR data from descent trajectories," in Proceedings of the 8th International Congress on Research in Air Transportation (ICRAT). Castelldefels, Catalonia: Eurocontrol/FAA, Jun 2018.

[17] A. J. Cook and G. Tanner, "European airline delay cost reference values: Updated and extended values," Tech. Rep., 2015. 